\title{
Looking to an anti-ageist future
}

\author{
The consequences of ageism have never been more apparent than in the context of a global pandemic. In this special \\ issue on ageism, Nature Aging demonstrates its commitment to supporting efforts to combat ageism in all its forms.
}

ervasive and insidious are words often used to describe ageism. The term was first coined in 1969 by Robert N. Butler, a renowned leader in the field of gerontology and the first director of the National Institute of Aging. He originally defined ageism as a "process of systematic stereotyping or discrimination against people because they are old", and likened it to racism and sexism, other forms of discrimination that manifest as prejudicial attitudes and practices against a group of people on the basis of a single characteristic. Butler also pointedly described (and perhaps foreshadowed) the serious consequences of such attitudes, stating that "ageism allows the younger generations to see older people as different than themselves; thus, they subtly cease to identify with their elders as human beings" (Butler, R. Why Survive? Being Old in America; Harper \& Row, 1975).

Ageism negatively affects many aspects of older people's lives but also has broader detrimental consequences for society. The global pandemic has brought some of these problems to the fore of our collective consciousness, thus creating an opportunity to galvanize action to address them. To help raise awareness about these issues and better understand what ageism is and how it can be combatted, this month's review and opinion section of the journal is focused on ageism. As the articles in this issue will attest, discrimination on the basis of age is evident in many settings, including health and long-term care settings, scientific research, the workplace and the broader community, in the form of socially entrenched negative attitudes surrounding the rights and capabilities of older people.

During the initial outbreak of the COVID-19 pandemic, the world witnessed first-hand what many people who work in aged care or related fields already knew and have been trying to raise the alarm about for years, even decades. Long-term care systems all over the world that were already badly under-resourced and under-staffed were completely overwhelmed by the pandemic. In their Correspondence, Stratton and colleagues use the experience in Canada as an example to argue that pre-existing apathetic attitudes towards older adults contributed to the deadly impact of the virus on long-term care residents.
In addition to long-term care settings, there are many other aspects of the way in which healthcare systems operate that either directly or indirectly discriminate against older adults and prevent them from receiving equal care and treatment. In a Comment, Sharon Inouye describes the forms of bias and discriminatory practices that occur in clinical settings, such as assumptions made during routine care for older adults in general practice and hospitals, and their exclusion from clinical trials. While these may often be subtle, unintended and implicit forms of bias, the consequences for health outcomes are significant and point to the need for improved clinical training.

One of the most commonly known and sometimes quite explicit forms of ageism is age discrimination in the workplace. What is less well known, however, is the extensive economic cost of such behavior. The failure to support an older and intergenerational workforce can result in missed opportunities for businesses and societies. In a World View, Joo Yeoun Suh of the American Association of Retired Persons (AARP), a non-profit organization aimed at empowering people to choose how they live as they age, discusses their recently published report on the cost of age discrimination in the workplace. That cost was estimated at US\$850 billion in gross domestic product (GDP) and US $\$ 545$ billion in lost wages and salaries in the USA in 2018 alone. Putting a financial bottom line on ageism will hopefully help to incentivize the large-scale changes that are needed from various stakeholders.

Although aging is universal, the experience for older adults and circumstances in which they age and are cared for can vary drastically between regions and countries of the world. In a World View, Alex Kalache, president of International Longevity Centre-Brazil, discusses ageism in the context of the Global South and the high potential for older adults to experience cumulative discrimination and inequality. He calls for individuals and societies to become anti-ageist: that is, to be proactive in trying to protect the rights and improve the lives of older people.

This sentiment is echoed in another World View by the World Health
Organization ahead of the publication of the first ever global report on ageism in March, and as part of their call to action to join the Global Campaign to Combat Ageism (http://bit.ly/3iWONy9). It is encouraging that a major focus of the UN Decade of Healthy Ageing (http://bit.ly/2Yn10D1) will be on combating ageism, and that this will not only lead to greater awareness of the issue, but also to new research, policies and actions.

Despite the increased awareness of explicit and implicit ageism and its potential negative impacts, a vast gap in evidence-based research remains. There is a serious need to improve our understanding of how ageist biases are formed or influenced, and their relationship to anti-social behaviors. Such behaviors include elder abuse or mistreatment, which are defined as intentional actions and inactions that cause harm, or create a serious risk of harm, to an older person by someone in a positon of trust (for example, a family member or a caregiver). In a Perspective, Pillemer and colleagues explore this topic in detail and lay out a framework for how future research should investigate the potential connection between ageism and elder mistreatment, and from there inform the development of appropriate interventions.

What this series of articles demonstrates is that on the one hand, for every instance of discrimination towards older adults, there are people and institutions willing to stand up for the rights of older adults, wherever ageism may manifest. But on the other hand, despite growth in awareness and advocacy, there is still an acute need to translate words into actions and accomplish societal change at scale. Nature Aging is committed to supporting these efforts and to help oppose ageism in all its forms, in research and in various societal contexts. Thus, we welcome impactful submissions that aim to better understand ageism or intervene on it, but also studies that are designed to address ageist biases in health research. Ultimately, the responsibility for tackling ageism and creating an anti-ageist future rests with each of us.

Published online: 11 February 2021 https://doi.org/10.1038/s43587-021-00038-2 